\title{
ORIGINAL
}

\section{LA EDUCACIÓN PARA LA SALUD EN LOS MANUALES ESCOLARES ESPAÑOLES (*)}

\author{
Valentín Gavidia Catalán
}

Departamento Didáctica CC. Experimentales y Sociales, Universidad de Valencia.

\section{RESUMEN}

Fundamento: El manual escolar, o libro de texto, es el instrumento educativo más utilizado por el profesorado. El objetivo de este trabajo es conoce los textos de la enseñanza primaria y secundaria. Es de suma importancia para averiguar su implantación en las aulas, así como ofrecer puntos de reflexión para los autores y editores de los textos y para el profesorado y el personal sanitario que asiste a las escuelas.

Método: El análisis se ha efectuado en 297 libros de texto, de los que 123 corresponden a la educación primaria, 149 a la secundaria obligatoria y 25 al bachillerato. El estudio atiende a cinco aspectos: 1) Presencia o ausencia de la Educación para la Salud; 2) Temas de salud considerados; 3) Nivel de salud que desarrollan; 4) Incardinación en la asignatura o tratamiento transversal, y 5) Aspectos metodológicos. Para cada uno de estos aspectos se han definido una serie de criterios de análisis que permiten contemplar su nivel de desade criterio.
rrollo.

Resultados: El 63\% de los libros analizados presentan temas relativos a la Educación para la Salud. Los temas más tratados se refieren a cuestiones de higiene (39,5\%), seguidos de la alimentación $(26,5 \%)$. El concepto de salud más presente es el de ausencia de $(26,5 \%)$. El concepto de salud más presente es el de ausencia de
enfermedad (36,9\%) y el de estado de bienestar (20\%). Algo más de enfermedad $(36,9 \%)$ y el de estado de bienestar (20\%). Algo más de
la mitad de los textos que tratan la salud ofrecen actividades de aprendizaje, aunque puntuales y aisladas $(57,7 \%)$. La metodología que más utilizan es la exposición informativa $(32,4 \%)$, sin atender los contenidos procedimentales ni a criterios actitudinales.

Conclusiones: Los actuales manuales escolares no constituyen un referente suficiente ni un recurso adecuado para que el profesorado desarrolle la Educación para la Salud en el aula, ya que no le prestan atención suficiente, a pesar de ser considerada materia transversal y, cuando lo hacen, sus orientaciones no movilizan actitudes y conductas.

Palabras clave: Educación en Salud. Promoción de la Salud. Salud escolar. Currículum. Libros de texto.

\section{Correspondencia:}

Valentín Gavidia Catalán

Dpto. Didáctica Ciencias Experimentales y Sociales, Universidad de Valencia

Correo electrónico: Valentin.Gavidia@uv.es

\section{ABSTRACT}

\section{Health Education in the Spanish School Manuals}

Background: The school manual or textbook is the educational tool most used by the teaching staff. This study is aimed as ascertaining how Education for Health is dealt with in Primary and Secondary school textbooks, which is of utmost importance as regards finding out how they are implemented in the classroom, as well as setting out some points for thought for the authors and editors of these texts and for the teaching staff and healthcare personnel providing assistance to the schools.

Method: A total of 297 textbooks were analyzed, 123 of which were used at the Primary level, 149 at the Compulsory Secondary level and 25 at the Higher Secondary level. This study deals with five aspects: 1) Presence or absence of Education for Health 2) Healthrelated topics included 3) Degree of health covered 4) Included in the subject or dealt with on a transversal basis and 5) Methodological aspects. A number of criteria for analysis have been defined for each one of these aspects which will make it possible to consider the degree to which they are covered.

Results: Sixty-three percent of the books analyzed included topics related to Education for Health. The topics dealt with most were related to matters of hygiene $(39.5 \%)$, followed by eating $(26.5 \%)$. The health-related concept most often dealt with is that of being disease-free $(36.9 \%)$ and a state of well-being (20\%). Somewhat over half of the textbooks dealing with health provide learning activities, although only in certain isolated cases $(57.7 \%)$. The methodology most often used is informative explanation $(32.4 \%)$ without dealing with the procedure-related contents or attitude-related criteria.

Conclusions: The current school manuals are not sufficient either as a point of reference or as an adequate resource in order for the teaching staff to provide Education for Health in the classroom, as they do not place sufficient emphasis thereon despite its being considered a transversal subject, and when they indeed do so, the way in which they are oriented does not set attitudes or behaviors into motion.

Key words: Health promotion. Health education. School health. Curriculum. Textbooks.

(*) El presente trabajo forma parte del Proyecto de Investigación titulado «Análisis del tratamiento de la Prevención de la Drogadicción y de la Educación para la Salud en los libros de texto españoles» financiado por el Instituto de Investigación de Drogodependencias (INID) de la Universidad Miguel Hernández. 


\section{INTRODUCCIÓN}

Existe consenso acerca del importante papel de la Promoción de la Salud y en concreto de las acciones de Educación para la Salud desarrolladas en la escuela, para la formación integral del alumnado. Los comportamientos espontáneos no aseguran el máximo de salud de las personas, de ahí la necesidad de que la educación formal obligatoria incorpore la salud entre sus objetivos. Esta promoción de la salud a través de la educación escolar, tiene especial importancia por los siguientes $\operatorname{motivos}^{1}$ : A) La niñez y la adolescencia son los momentos más apropiados para acoger y adaptar nuevos modelos de vida. B) Todas las personas pasan a lo largo de su vida una etapa en la escuela, por lo que es la institución a la que la sociedad confía la socialización, es decir, la transmisión de la cultura, los conocimientos y los valores por los que se rige. C) Es el lugar apropiado para preparar a los ciudadanos para la vida, formarlos para que sean capaces de tomar, de manera razonada e informada, las decisiones que van a tener consecuencias claras sobre su salud y la de los que les rodean. D) Ayuda a los ciudadanos a saber interpretar críticamente, las condiciones de vida con el fin de aprender a leer y comprender las condiciones que producen enfermedad y las soluciones para poder superarlas. E) Es un ambiente de trabajo que puede, y debe permitir al alumnado vivir situaciones favorables para promover la salud y evitar el malestar o la enfermedad. F) Favorece la comunicación entre todos los componentes del sistema educativo (alumnado, profesorado, familiares, personal no docente, etc.) y los agentes sociales. G) Constituye un elemento de presencia imprescindible en las escuelas dado que existe una considerable evidencia que demuestra que la mala salud inhibe el aprendizaje, existiendo una estrecha relación entre la conducta orientada hacia la salud y los resultados educativos ${ }^{2}$.

La mayoría de nuestros comportamientos los aprendemos en los primeros años de nuestra vida, años que pasamos en la escuela, que es el lugar que la sociedad destina para la transmisión de la cultura. Es obvio, por lo tanto, que el sistema educativo y la sociedad en su conjunto no desaprovechen esta oportunidad para contribuir a la formación de estilos de vida saludables en la población.

A esto hay que añadir que en los últimos años han surgido una serie de demandas sociales que empujan por introducir como metas del sistema educativo unos objetivos a los que se estaba ajeno. Se ha visto la urgencia en dar respuesta, también desde la enseñanza, a problemas y preocupaciones que forman parte de la realidad de la vida cotidiana $^{3}$. Entre estos problemas se encuentran los relacionados con la salud y los estilos de vida: el consumo de drogas, la violencia, el sida, las enfermedades cardiovasculares, etc. Ante esta realidad se impone potenciar un tipo de educación que favorezca la autonomía, la capacitación para la vida y que facilite la autoprotección frente a los riesgos y el autocontrol como preparación eficaz para una mejor integración social. Pero la escuela poco puede hacer si no dispone de un profesorado preparado y de los recursos necesarios para llevar adelante una acción que responda a las necesidades de los educandos y de la sociedad.

El objetivo del presente trabajo consiste en analizar el tratamiento que ofrecen los libros de texto a la Educación para la Salud, bien entendido que no se trata de libros específicos de este tema sino de los textos que los escolares estudian en las materias curriculares de la enseñanza obligatoria y el bachillerato. Sabemos que el recurso educativo más generalizado es el libro de texto, por lo que nuestro propósito permite aproximarnos a la realidad de la Educación para la Salud en la escuela.

La importancia de este estudio es obvia ya que los libros de texto constituyen el punto de referencia más cercano que tiene el profe- 
sorado para su trabajo profesional; en ellos se concretan los diversos modelos de enseñanza-aprendizaje de los temas de salud; ofrecen una muestra de los conceptos que se utilizan; son materiales confeccionados por profesores; y constituyen un indicador de la cultura que se desarrolla en el aula.

A todo ello hay que añadir que el profesorado, a lo largo de su formación académica, no ha tenido una formación específica en estos temas, no se le ha ofrecido ninguna pauta de actuación en el aula sobre esta problemática, y tiene al libro de texto como recurso más utilizado. Todo lo cual convierte a los manuales escolares en pieza clave para aproximarnos a conocer la situación de la enseñanza-aprendizaje en general, y en particular de la Educación para la Salud en la escuela.

\section{MATERIAL Y MÉTODOS}

Se han analizado 297 textos pertenecientes al actual Sistema Educativo (LOGSE), de los cuales 123 corresponden a la etapa Primaria, 149 a la Secundaria y 25 al Bachillerato. La muestra comprende textos de todas las asignaturas y de todos los cursos de los niveles de primaria, secundaria obligatoria (ESO) y bachillerato.

El estudio se ha efectuado atendiendo a cinco aspectos ${ }^{4}:$ 1) la presencia o ausencia de temas relativos a la Salud, 2) los temas de salud considerados, 3) los niveles de representación del concepto de salud, 4) el tratamiento transversal, y 5) los aspectos metodológicos.

Criterio 1. Presencia o ausencia de temas relativos a la Salud. Se valora la presencia o ausencia de temas que, de alguna forma, están relacionados con la salud. No hablamos de Educación para la Salud sino de salud en general, por lo que se entiende como afirmativo el tratamiento de temas como anatomía, fisiología, vacunas, enfer- medades, etc., así como el cuerpo humano, los cuidados personales, las relaciones personales, el consumismo, etc., de forma que el hecho de aparecer algunas de estas cuestiones a lo largo de los capítulos que componen el texto, se considera como presencia positiva. Este criterio es la llave que abre el estudio posterior, pues luego se analizan únicamente los textos que aquí dan positivo.

Criterio 2. Los temas de salud considerados. En este criterio atendemos a los cuatro grandes grupos en los que hemos dividido la problemática de la Educación para la Salud: Cuidado personal (higiene, vacunas, contagios, etc.), la sociedad de consumo (publicidad, modas, etc.), equilibrio personal (sexualidad, drogas, etc.) y alimentación y nutrición (nutrientes, dietas, anorexia, etc.).

Criterio 3. Los niveles de representación del concepto de salud. Una representación adecuada de la salud permite plantear actividades didácticas con el enfoque necesario para que los estudiantes puedan realizar su propio aprendizaje y llegar a traducir sus conocimientos en comportamientos saludables. Los cinco niveles de representación considerados son los siguientes ${ }^{5}$ :

- Nivel I: Concepción tradicional: «La salud es la ausencia de enfermedades e invalideces» o bien es «la vida en el silencio de los órganos». Dimensión estrictamente física $^{6}$. Sinónimo de vida.

- Nivel II: «El estado de completo bienestar físico, mental y social y no solamente la ausencia de enfermedades». La salud como estado de bienestar e ideal de vida. A la dimensión física se le añade la psíquica y social $^{7}$.

- Nivel III: «La salud es el estado de adaptación al medio y la capacidad de funcionar en las mejores condiciones en dicho medio». La salud como equilibrio con el entorno. El locus control es externo al individuo ${ }^{8}$. 
- Nivel IV: «La salud es aquella manera de vivir que es autónoma, solidaria y profundamente gozosa». La salud como determinados comportamientos y prohibiciones. El locus control es interno a la persona ${ }^{9}$.

- Nivel V: «La salud es la capacidad de desarrollar el propio potencial personal y responder de forma positiva a los problemas del ambiente». La salud holística o global, como proceso multicausal que se identifica con el desarrollo personal y social, y como medio para la mejora de la calidad de vida ${ }^{10}$.

Criterio 4. Consideración de la transversalidad. La Educación para la Salud forma parte de lo que se denominan materias transversales, por lo que deben aparecer en los libros de texto de todas las materias, pero esto lo pueden hacer con diferentes niveles de integración ${ }^{11}$ :

T.1. No se llevan a cabo actividades específicas de Educación para la Salud. Consideramos este nivel cuando en el libro aparecen informaciones acerca de temas relativos de la salud pero no presentan ningún tipo de actividades a realizar.

T.2. Realización de actividades puntuales. Presenta algunas actividades, pero lo hace de manera puntual, aislada y casi marginal del desarrollo de la propia materia. Suele aparecer en las páginas finales de algunos temas.

T.3. Tratamiento vertical o disciplinar. La Educación para la Salud es apreciada como una disciplina o parte de ella. Es el caso de asignaturas optativas, o cuando alguna materia como las ciencias naturales o el conocimiento del medio desarrolla estos temas con las mismas características que el resto. Suele darse en cuestiones como el cuerpo humano y su fisiología, donde es frecuente tratar aspectos de higiene y prevención de enfermedades.

T.4. Tratamiento transversal desde las áreas de conocimiento. La Educación para la
Salud colabora en la construcción de los contenidos de la propia materia y su información y actividades se encuentran entrelazadas con el resto del texto. No significa una ampliación de los contenidos, sino un cambio metodológico en el que la salud impregna el acto didáctico y donde se pueda conjugar el saber escolar con el saber cotidiano.

Criterio 5. Metodología utilizada. Se ha tenido especial cuidado en este apartado ya que su tratamiento evidencia la fundamentación psicopedagógica de autores o editores. Hemos establecido la siguiente tipología ${ }^{12}$ :

M.1. Ofrece simplemente información. Sigue un modelo tradicional expositivo de enseñanza y de aprendizaje memorístico, creyendo que una información de hechos y conceptos puede producir cambios de actitudes y conductas.

M.2. Favorece el aprendizaje a través del cambio conceptual. Las personas construyen el conocimiento a partir de sus propias representaciones, vivencias y relaciones con el mundo que les rodea. En los textos se tendrá en cuenta sus ideas, competencias y experiencias sin basarse exclusivamente en contenidos expositivos; etc.

M.3. Procura el cambio conceptual y metodológico. Se aprende cuando se le da significado al objeto del conocimiento y cuando éste es relevante. Por ello es importante que los libros de texto justifiquen el tratamiento de los problemas de salud presentando la necesidad de su estudio y que las cuestiones elegidas se aborden a partir de las competencias del alumnado a quien se dirigen. Así pues, las estrategias de enseñanza deben basarse en un planteamiento de situaciones problemáticas.

M.4. Potencia el cambio actitudinal, necesario para un aprendizaje significativo y condición necesaria, aunque puede que no suficiente, para adquirir un determinado comportamiento. Estudiamos si los textos presentan 
problemas de salud concretos y sentidos por el alumnado, si se proponen debates y otras actividades en las que se ponen en juego visiones, actitudes y valores, etc.

M.5. El entorno cobra importancia. No se trata de educar sólo la autorresponsabilidad de cada alumno sino de analizar de manera crítica la influencia del medio en el pensamiento y comportamiento humano. Una actitud positiva, aún siendo un factor básico de la conducta, no asegura un comportamiento deseable respecto a la salud. (Green y Kreutter 1991). Estudiamos la atención a la familia, a la escuela, a los amigos, los medios de comunicación, la publicidad, etc.

M.6. El alumnado como agente activo de salud. La participación del alumnado en la transformación de su entorno es una estrategia metodológica que debe ser asumida por el profesorado y por los libros de texto. Debemos pasar de una concepción de Escuela Saludable a la de Escuela Promotora de la Salud. Los textos presentarán la utilidad de lo estudiado, haciendo especial hincapié en su funcionalidad, en la necesidad de colaborar en la mejora del medio y presentando actividades para este cambio.

\section{RESULTADOS}

\section{Primer criterio: Presencia de la Educación para la Salud}

Los resultados del estudio nos indican que el $63 \%$ de los textos analizados tratan cuestiones relacionadas con la salud, mientras que el $37 \%$ no lo hace. Este porcentaje se mantiene prácticamente igual a lo largo de las tres etapas educativas como podemos observar en la tabla 1 y figura 1 .

Estos resultados los podemos desglosar atendiendo a la presencia en las diferentes materias y entonces observamos que ciencias naturales es la asignatura donde los temas de salud aparecen con más frecuencia, seguidos de los libros de lengua y de ciencias sociales. Destacamos que también aparecen cuestiones relativas a la salud en los libros de matemáticas.

\section{Segundo Criterio: Contenidos de Salud en los libros de texto}

Los temas de salud se han agrupado en cuatro grandes bloques temáticos. Los resultados vienen expresados en la tabla 2 , y en ella observamos que el bloque 1 (higiene, enfermedades infecciosas, vacunas, sida, etc.) es el más representado en los libros de texto $(39,5 \%)$, seguido de los temas de alimentación $(26,5 \%)$. Los temas de consumo (moda, publicidad, etc.) y equilibrio personal (drogodependencias, estilos de vida, sexualidad, etc.) son menos tratados $(18,3 \%$ y $15,8 \%$ respectivamente).

La distribución de estos temas se mantiene prácticamente constante a lo largo de las etapas del Sistema Educativo a excepción de los contenidos del bloque 3, Equilibrio personal, que pierde importancia en el Bachillerato, pero lo ganan los temas relativos al Consumo y sobre todo la Higiene y las enfermedades (sida).

\section{Tercer criterio: Niveles de representación del concepto de salud}

Los resultados de observar el nivel de representación del concepto de salud que aparece en los libros de texto se recogen en la tabla 3. El primer dato a destacar es que el nivel de la representación de salud decrece a medida que aumenta la edad de los alumnos a los que se destinan los textos. Así, mientras en Primaria el nivel de representación sobre un máximo de 5 es de 2,54, en el bachillerato es de 1,8 . Es decir, el nivel de representación de la salud es superior en los textos de primaria, que en los de secundaria, siendo los del bachillerato los que presentan un menor nivel. La concepción más baja, $\mathrm{N}-1$, es la más usada y se encuentra en el 36,9\%, 
Tabla 1

Presencia de temas de salud en los textos de la LOGSE

\begin{tabular}{|c|c|c|c|c|c|}
\hline \multirow{2}{*}{ Etapas } & \multicolumn{2}{|c|}{ Presencia } & \multicolumn{2}{|c|}{ Ausencia } & \multirow{2}{*}{$\frac{\text { Total }}{\text { Libros analizados }}$} \\
\hline & $N$ & $\%$ & $N$ & $\%$ & \\
\hline Primaria & 77 & $62.6 \%$ & 46 & $37.4 \%$ & 123 \\
\hline Secundaria & 92 & $61.3 \%$ & 57 & $38.7 \%$ & 149 \\
\hline Bachillerato & 18 & $72 \%$ & 7 & $28 \%$ & 25 \\
\hline Total & 187 & $62,8 \%$ & 110 & $37,2 \%$ & 297 \\
\hline
\end{tabular}

Figura 1

Presencia de los temas relativos a la Salud en los textos de la LOGSE

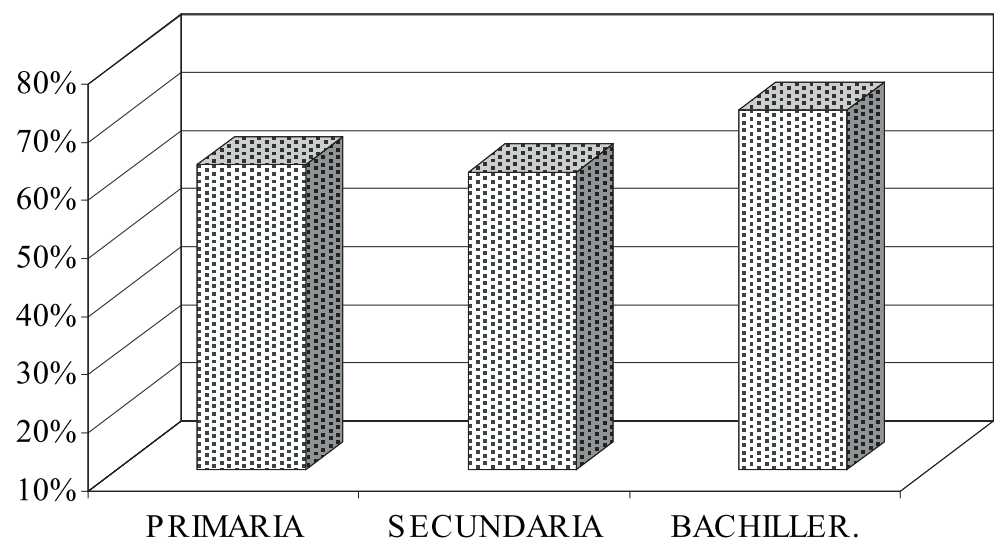

Tabla 2

Contenidos de Salud en los textos de la LOGSE

\begin{tabular}{|lccccccccc|}
\hline \multirow{2}{*}{ Etapas } & \multicolumn{2}{c}{$\begin{array}{c}\text { BLOQUE 1 } \\
\text { (Higiene) }\end{array}$} & \multicolumn{2}{c}{$\begin{array}{c}\text { BLOQUE 2 } \\
\text { (Consumo) }\end{array}$} & \multicolumn{2}{c}{$\begin{array}{c}\text { BLOQUE 3 } \\
\text { (Equilibrio) }\end{array}$} & \multicolumn{2}{c|}{$\begin{array}{c}\text { BLOQUE 4 } \\
\text { (Alimentación) }\end{array}$} & TOTAL \\
\cline { 2 - 10 } & $N$ & $\%$ & $N$ & $\%$ & $N$ & $\%$ & $N$ & $\%$ & Libros analizados \\
\hline Primaria & 62 & $40.5 \%$ & 27 & $17.6 \%$ & 22 & $14.4 \%$ & 42 & $27.5 \%$ & 153 \\
Secundaria & 52 & $37.5 \%$ & 24 & $17.7 \%$ & 26 & $19.1 \%$ & 35 & $25.7 \%$ & 137 \\
Bachillerato & 10 & $47.6 \%$ & 5 & $23.8 \%$ & 1 & $4.8 \%$ & 5 & $23.8 \%$ & 21 \\
Total & 124 & $39.5 \%$ & 56 & $18.3 \%$ & 49 & $15.8 \%$ & 82 & $26.5 \%$ & 311 \\
\hline
\end{tabular}

lo que indica que todavía la idea de salud está muy medicalizada, pero el conjunto de textos que consideran aspectos relativos al entorno, al estilo de vida o al desarrollo social es superior al $41 \%$.

\section{Cuarto criterio: Tratamiento transversal}

Los resultados de aplicar a los libros de texto los criterios de observación de la transversalidad se presentan en la tabla 4. La suma de 
Tabla 3

Niveles de representación del concepto de salud en los textos de la LOGSE

\begin{tabular}{|lcccccccc|}
\hline \multirow{2}{*}{ Niveles de representación } & \multicolumn{2}{c}{ Primaria } & \multicolumn{2}{c}{ Secundaria } & \multicolumn{2}{c|}{ Bachillerato } & \multicolumn{2}{c|}{ Total } \\
\cline { 2 - 9 } & $N$ & $\%$ & $N$ & $\%$ & $N$ & $\%$ & $N$ & $\%$ \\
\hline N-1. Ausencia. & 23 & $30 \%$ & 34 & $37 \%$ & 12 & $66.7 \%$ & 69 & 36,9 \\
N-2. Bienestar. & 18 & $23.4 \%$ & 19 & $20.6 \%$ & 0 & $0 \%$ & 37 & 19.7 \\
N-3. Entorno. & 12 & $15.6 \%$ & 25 & $27.2 \%$ & 5 & $27.7 \%$ & 42 & 22.4 \\
N-4. Estilo de vida. & 19 & $24.6 \%$ & 7 & $7.6 \%$ & 0 & $0 \%$ & 26 & 14 \\
N-5. Desarrollo personal y social. & 5 & $6.4 \%$ & 7 & $7.6 \%$ & 1 & $5.6 \%$ & 13 & 5.3 \\
Total & 77 & & 92 & & 18 & & 187 & 100 \\
Nivel Medio & & 2,54 & & 2,24 & & 1,8 & & 2,34 \\
\hline
\end{tabular}

\section{Figura 2}

Niveles de representación del concepto de salud en los textos de la LOGSE

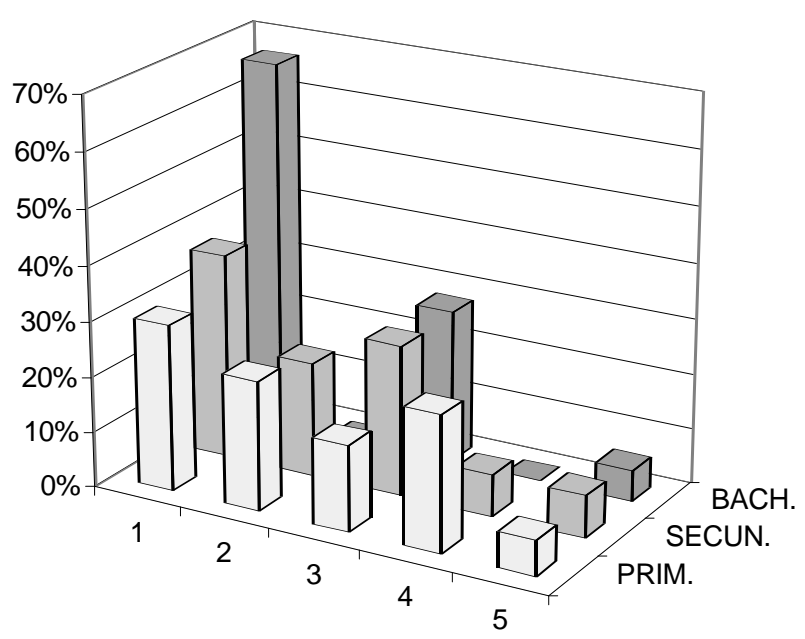

las frecuencias que aparece en los diversos apartados puede ser superior a $100 \%$ debido a que los textos pueden considerar la EpS de varias formas al mismo tiempo, esto es, presentar un capítulo íntegro dedicado a ella y al mismo tiempo, ofrecer en otros capítulos informaciones, curiosidades o ejemplos de aplicación de cuestiones relativas a la salud.

El primer resultado que llama la atención es comprobar que un $27,3 \%$ de los libros que tratan cuestiones de salud no presentan actividades específicas de esta temática, y el
$57,7 \%$ lo hacen sólo de manera puntual, unas veces aisladas de los contenidos de la materia y otras como mera ilustración del tema donde se encuentran. Algo más del 50\% de los libros que tratan la salud lo hacen como un capítulo concreto, bien en exclusiva, bien formando parte de otro más amplio, pero sin establecer relaciones con otros capítulos, por lo que consideramos que su tratamiento es disciplinar y en modo alguno transversal. Son muy pocos $(12,3 \%)$ los textos que tratan la salud desde el punto de vista transversal que hemos expuesto. 
Tabla 4

Transversalidad en los textos de la LOGSE

\begin{tabular}{|lccccccccc|}
\hline \multirow{2}{*}{ Tratamiento de la transversalidad } & \multicolumn{2}{c}{ Primaria } & \multicolumn{2}{c}{ Secundaria } & \multicolumn{2}{c|}{ Bachillerato } & \multicolumn{3}{c|}{ Total LOGSE } \\
\cline { 2 - 10 } & $N=77$ & $\%$ & $N=92$ & $\%$ & $N=18$ & $\%$ & $N=187$ & $\%$ \\
\hline $\begin{array}{l}\text { T1. No presenta actividades } \\
\text { específicas }\end{array}$ & 30 & 39 & 19 & 20.6 & 2 & 11.1 & 51 & 27.3 \\
$\begin{array}{l}\text { T2. Presenta actividades puntuales } \\
\text { y aisladas }\end{array}$ & 41 & 53.2 & 52 & 56.5 & 15 & 83.3 & 108 & 57.7 \\
$\begin{array}{l}\text { T3. Tratamiento disciplinar o vertical } \\
\text { T4. Tratamiento transversal desde } \\
\text { el área }\end{array}$ & 49 & 63.6 & 38 & 41.3 & 7 & 41 & 94 & 50.3 \\
\hline
\end{tabular}

Figura 3

Transversalidad de la Educación para la Salud en los textos de la LOGSE

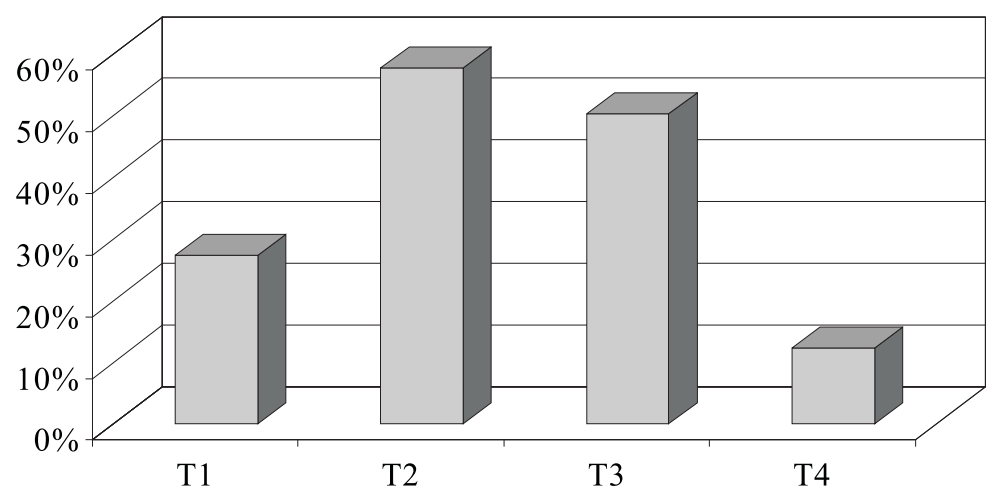

\section{Quinto criterio: los aspectos metodológicos}

Los resultados de aplicar los criterios que caracterizan una metodología de la Educación para la Salud en el análisis de libros de texto se recogen en la tabla 5. La metodología utilizada va perdiendo complejidad a medida que aumenta la edad de los alumnos a los que los textos van destinados, pues de presentar un nivel medio de 3 en la etapa primaria se pasa a 2,2 en la secundaria y a 1,7 en el bachillerato, de un máximo de 6 puntos.

Los textos de primaria de ciencias naturales realizan, en líneas generales, un trata- miento metodológico bastante correcto, ya que no sólo ofrecen información, sino que tienen en cuenta otros criterios necesarios para procurar un cambio metodológico, procedimental y actitudinal en el alumnado, como son la toma de decisiones, los juegos, etc., así como un análisis del entorno para acometer, en lo posible, una mejora en los factores que condicionan determinadas pautas de conducta. No obstante, no son los únicos que tienen en cuenta estos criterios ya que encontramos libros de otras materias como las ciencias sociales, la lengua o la religión, que también los poseen.

Los buenos criterios metodológicos que se dan en los textos de primaria decaen cuan- 
Tabla 5

Metodología de la Educación para la Salud en los textos de la LOGSE

\begin{tabular}{|c|c|c|c|c|c|c|c|c|}
\hline \multirow{2}{*}{ Metodologia de la Educación para la Salud } & \multicolumn{2}{|c|}{ Primaria } & \multicolumn{2}{|c|}{ Secundaria } & \multicolumn{2}{|c|}{ Bachillerato } & \multicolumn{2}{|c|}{ Total LOGSE } \\
\hline & $N$ & $\%$ & $N$ & $\%$ & $N$ & $\%$ & $N$ & $\%$ \\
\hline M1. Información sobre salud. & 15 & $19.5 \%$ & 35 & $38.7 \%$ & 10 & $55.5 \%$ & 60 & $32.4 \%$ \\
\hline M2. Se atiende al cambio conceptual. & 23 & $30 \%$ & 29 & $31.2 \%$ & 6 & $33.3 \%$ & 58 & $30.8 \%$ \\
\hline $\begin{array}{l}\text { M3. Se procura el cambio conceptual y } \\
\text { metodológico. }\end{array}$ & 8 & $17 \%$ & 13 & $14 \%$ & 1 & $5.6 \%$ & 22 & $11.7 \%$ \\
\hline $\begin{array}{l}\text { M4. El cambio actitudinal, base del } \\
\text { comportamiento. }\end{array}$ & 13 & $19.5 \%$ & 8 & $8.6 \%$ & 0 & $0 \%$ & 21 & $11.2 \%$ \\
\hline $\begin{array}{l}\text { M5. El entorno social cobra importancia de } \\
\text { conductas. }\end{array}$ & 15 & $40 \%$ & 1 & $1.1 \%$ & 1 & $5.6 \%$ & 17 & $9 \%$ \\
\hline $\begin{array}{l}\text { M6. El alumnado como agente activo de } \\
\text { salud. }\end{array}$ & 3 & $4 \%$ & 6 & $6.4 \%$ & 0 & $0 \%$ & 9 & $4.9 \%$ \\
\hline Total & 77 & & 92 & & 18 & & 187 & 100 \\
\hline Nivel Medio & 3 & & 2,2 & & 1,7 & & & \\
\hline
\end{tabular}

Figura 4

Metodología de la Educación para la Salud en los textos de la LOGSE

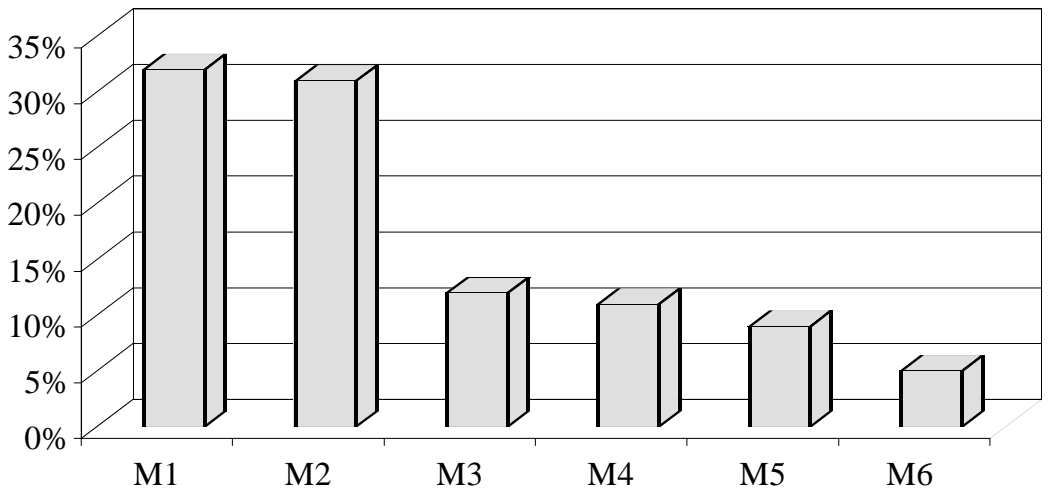

do se analizan los de secundaria, en los que sólo los de biología presentan actividades dirigidas a algo más que a ofrecer información sobre la materia de estudio ya que procuran un cambio de actitudes y la modificación de conductas. El resto de las materias, en general, no dedican el esfuerzo necesario para acercarse a estos objetivos conductuales. Este aspecto se agudiza todavía más en los textos escritos para el bachillerato.

\section{DISCUSIÓN}

Se han diseñado unos criterios para analizar el tratamiento que ofrecen los libros de texto a la Educación para la Salud, y se han aplicado a 297 manuales escolares publicados según la normativa desarrollada por la nueva ley que regula el sistema educativo español (LOGSE, 1990). 
En el capítulo anterior de resultados hemos visto, en líneas generales, lo siguiente: los libros de texto de las etapas inferiores dan más importancia a los temas de salud que los de las etapas superiores, ya que sus contenidos los desgranan por todo el texto; los que más se implican en el tratamiento de la Educación para la Salud son los que desarrollan las asignaturas de ciencias naturales/conocimiento del medio; ningún libro de texto presenta la Educación para la Salud con carácter interdisciplinar; y los niveles de integración en el resto de la materia son muy bajos.

Junto con la importancia que estos resultados pueden representar para ser tenidos en cuenta por el profesorado, los autores de textos, las editoriales y las administraciones educativa y sanitaria, queremos señalar las dificultades que un estudio de estas características comporta y hacer patente las limitaciones que los autores de los textos tienen para introducir ciertos cambios o innovaciones, al estar sujetos a líneas editoriales, visiones de mercado, etc.

Existe dificultad en atribuir a determinados contenidos el calificativo de Educación para la Salud. Aunque es evidente la diferencia que existe entre los temas de anatomía o fisiología y los de Educación para la Salud, sin embargo, en ciertos niveles educativos, aquéllos son necesarios para comprender los segundos y así poder abordar cuestiones comportamentales. Así, el primer eslabón para que se pueda dar un cambio de actitudes es una suficiente base conceptual ${ }^{13}$, necesaria en el nivel educativo de secundaria, pero no tan fundamental en los niveles de primaria. Este problema sólo ocurre con los textos de biología, no así con los de lengua o sociales, por lo que entendemos que cuando en estos textos aparecen temas de salud se trata de Educación para la Salud. Nuestra propuesta para los textos de biología es que sus autores realicen el esfuerzo de ir más allá de la mera información biologicista y presenten los temas de Educación para la Salud enmar- cados en una problemática más cercana y cotidiana al alumnado.

También debemos considerar el siguiente hecho: a medida que los libros de texto tienen un nivel superior de dificultad, están más especializados y desarrollan con mayor profundidad la problemática que le es propia, lo que significa que la Educación para la Salud no suele aparecer entre sus páginas (la historia o las matemáticas del bachillerato no se «distraen» con estos temas). Esto debe hacernos pensar en los obstáculos de ciertos planteamientos de transversalidad, pues no en todas las materias ni en todos los niveles se pueden tratar cuestiones específicas de Educación para la Salud. Es cierto que cabe un tratamiento inespecífico y globalizador, pero ello comporta la dificultad de su identificación. Esto implica, para los niveles educativos superiores, entender la transversalidad como la presentación al alumnado de determinados momentos de aprendizaje o proyectos de trabajo en los que se aborden cuestiones de Educación para la Salud de interés para ellos, dado que éstas no aparecen en sus textos de aula.

Las concepciones holísticas de salud aparecen con mayor frecuencia en los libros de texto de niveles más inferiores, lo que en primera instancia parece normal, dado que se trata de libros más generalistas. Sin embargo, aquí se encuentra un reto que deben abordar los autores de los textos de niveles superiores y no limitarse a dar recomendaciones y prescripciones basadas únicamente en una fundamentación médica. Es importante no dar la impresión al alumnado de que la salud es algo parcial que consiste en una serie de prohibiciones (todo lo que nos gusta no es saludable). Presentar la salud como un instrumento que sirve para mejorar nuestra calidad de vida es algo que «vende» entre la población juvenil y puede ser utilizado en sus textos de estudio.

Así mismo, somos conscientes de las limitaciones que tienen los autores de los textos 
para ofrecer actividades que puedan incidir en aspectos actitudinales o comportamentales, y mucho más en presentar a la escuela como promotora de salud, a través de considerar al alumnado como agente o vehículo difusor del valor «salud». Dentro de las dificultades, esta actuación es posible para el profesorado que se encuentra en contacto directo con su alumnado, pues puede seleccionar y contextualizar los temas a tratar y utilizar los diversos elementos que intervienen en una acción de estas características, como es la familia, el grupo de amigos, el equipo de profesores, el personal sanitario y demás agentes sociales. Sin embargo, y a pesar de estas dificultades, quisiéramos esperar de los autores de texto que mencionen toda esta gama de posibilidades y muestren a sus lectores ideas y acciones bien fundamentadas a desarrollar fuera del aula, de forma que el tratamiento posterior de sus resultados en clase sirva para su aprendizaje conceptual, procedimental y actitudinal, al tiempo que desde la escuela se interviene en el entorno cercano para hacerlo cada vez más saludable.

Después del análisis efectuado podemos decir que los libros de texto actualmente vigentes en el mercado no constituyen un referente suficiente ni un recurso adecuado para que el profesorado desarrolle la Educación para la Salud con sus alumnos en la escuela, ya que no le prestan la atención conveniente, a pesar de ser considerada como materia transversal, y cuando lo hacen su nivel de representación del concepto de salud es bajo y sus orientaciones metodológicas tampoco van dirigidas a facilitar una movilización de actitudes y un cambio conductual.

Deseamos que los resultados de este estudio permitan una reflexión entre todos los agentes implicados en los manuales escolares para que superen una situación que, siendo mejor que la que existía con la Ley General de Educación del año 1970, dista todavía que desear.

\section{BIBLIOGRAFÍA}

1. Ferrari A. Finalidad y métodos de la Educación para la Salud en la escuela. Serie de conferencias recogidas en el Programa de Educación para la Salud. Comisión Mixta Sanidad-Educación. Valencia: Generalitat Valenciana; 1986.

2. Unión Internacional de Promoción y Educación para la Salud. La Evidencia de la Eficacia de la Promoción de la Salud. Madrid: Ministerio de Sanidad y Consumo; 2000.

3. Unión europea, Parlamento europeo y Consejo. Comunicado 1 de Junio 1994. 94/0130 (COD). Documento CB-CO-94-220-ES-C.

4. Gavidia V. Salud, educación y calidad de vida: De cómo las concepciones del profesorado inciden en la salud. Santa Fe de Bogotá: Magisterio; 1998.

5. Gavidia V. Criterios para analizar las representaciones del concepto de salud. Su aplicación en el profesorado. Comunicación en el V Congreso Internacional sobre Investigación en la Didáctica de las Ciencias y de las Matemáticas. Murcia. Enseñanza de las Ciencias, Número Extra, 1997; 141-142.

6. Leriche R. Chirurgie de la douleur. Pour une pédagogie de la santé. París: Masson \& Cie; 1937. [Recogido en Mémoire FOPES.UCL, Louvain-laNeuve, 1985].

7. Organización Mundial de la Salud. Carta Constitucional. Ginebra: Organización Mundial de la Salud; 1946.

8. Dubos RJ. Man adapting. New Haven: Yale University Press; 1965.

9. Polaino Lorente A. Educación para la Salud. Barcelona: Herder; 1987.

10. Organización Mundial de la Salud. Oficina Regional para Europa. Health Promotion. A discussion document on the concepts and principles. Copenhague: Organización Mundial de la Salud; 1986.

11. Gavidia V, Rodes MJ. Desarrollo de la Educación para la Salud y del Consumidor en los Centros Docentes. Madrid: Ministerio de Educación y Cultura-CIDE; 2000.

12. Gavidia V, Sieres J y Moliner E. La prevención de la drogadicción en los libros de texto. Aula de Innovación educativa 2000; $89: 21-9$.

13. Yus Ramos R. (1993) Las transversales: conocimiento y actitudes. Cuadernos de Pedagogía 1993; 217: 76-9. 